\title{
Diseño y validación de un cuestionario de propensión al accidente deportivo
} (PAD-22)

\section{Design and validation of a propensity to sports accident questionnaire} (PAD-22)

\section{Desenho e validação de um questionário de propensão ao acidente esportivo} (PAD-22)

\author{
Pedro Ángel Latorre Román y Antonio Pantoja Vallejo
}

Universidad de Jaén

\begin{abstract}
Resumen: El propósito de este estudio ha sido el diseño y validación de una escala de propensión al accidente deportivo en deportistas. La muestra

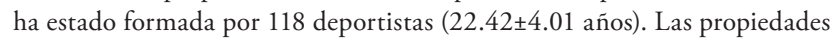
psicométricas de la escala se obtuvieron mediante un proceso de validez de contenido, concurrente con escala SSS-V y un análisis factorial de los componentes principales. Como resultados obtuvimos un cuestionario de 22 ítems formado por cinco factores y ajustado a los tópicos de validez y fiabilidad científica. Se encuentran diferencias significativas $(\mathrm{p}<.05)$ por deporte practicado y sexo en la escala PAD-22 y SSS-V.

Palabras clave: Accidente, deporte, riesgo, propensión.

Abstract: The purpose of this study was to design and validate a scale of the accident prone sports athletes. The sample has been formed by 118 athletes (22.42 \pm 4.01 years old). The psychometric properties of the scale were obtained through a content validity's process, concurrent validity with the scale SSS-V and factorial analysis of main components. As a result we
\end{abstract}

obtained a 22-item questionnaire formed by five factors and adjusted to scientific validity and reliability's topics. They found significant differences $(\mathrm{p}<.05)$ and gender in sport practiced on the scale PAD-22 and SSS-V. Keywords: Accident, sports, risk, propensity.

Resumo: O propósito desse estudo foi de desenhar e validar uma escala de propensão ao acidente esportivo em esportistas. A mostra foi formada por 118 esportistas $(22.42 \pm 4.01$ anos de idade). As propriedades psicrométricas da escala foram obtidas mediante um processo de validação do conteúdo, concorrente com a escala de SSS-V e um analise fatorial dos componentes principais. Como resultado obtivemos um questionário de 22 itens formado por cinco fatores e ajustado aos propósitos da validez e fiabilidade cientifica. Foram encontradas diferenças significativas $(\mathrm{p}<.05)$ por esporte praticado e sexo, na escala PAD-22 e SSS-V.

Palavras chave: Acidente, esporte, risco e propensão.

\section{Introducción}

Las actividades físicas y deportivas aportan un gran número de beneficios a la salud de las personas, no sólo a nivel fisiológico, sino también a nivel psicológico y social, pero representan en algunos casos un riesgo de sufrir algún tipo de lesión (Díaz, Buceta, y Bueno, 2004). En los últimos años se está produciendo un aumento importante en el número de lesiones que padecen los deportistas, principalmente a nivel aficionado. Este incremento puede venir provocado por los cambios socioeconómicos que ha experimentado nuestra sociedad, por el aumento del tiempo de ocio de que disponen los ciudadanos, por un cambio de actitud en el deporte de competición o por la mayor presión que se ejerce a nivel mediático sobre los deportistas, exigiéndoles en todo momento la victoria. Este último factor se extiende en muchos casos

Correspondencia: Pedro Ángel Latorre Román.

Universidad de Jaén. Paraje de las Lagunillas, s/n. Jaén,

Edificio D2. Despacho 142. Email: platorre@ujaen.es. al ciudadano de a pie, que asume que la victoria es el aspecto más importante de la práctica deportiva, aumentando su competitividad y llevándole a realizar conductas que pueden resultar peligrosas para su integridad física. Estudios epidemiológicos revelan que las lesiones han ido adquiriendo una gran importancia en el contexto deportivo, debido fundamentalmente al aumento de la participación (Olmedilla, Blas y Laguna, 2010; Olmedilla, García y Martínez, 2006). Como consecuencia de la importancia que este problema representa en el contexto deportivo, diversos estudios se han orientado hacia la identificación y el control de los diferentes factores que pueden incidir en el riesgo de los deportistas a lesionarse. El estudio de las lesiones y accidentes deportivos requiere un análisis multidimensional de diferentes aspectos, tanto físicos, como psicológicos y situacionales, y éstos se concretan en factores internos y externos. Dentro de los factores internos, se destacan los aspectos fisiológicos, biomecánicos, psicológicos, la edad, el deterioro del cuerpo, el historial de lesiones, 
la falta de preparación física para una determinada tarea, la falta de adherencia a medidas preventivas, una alimentación inadecuada, la fatiga, la realización de conductas de riesgo y el sobreentrenamiento. $Y$ en los factores externos, es preciso considerar las deficiencias en las condiciones ambientales, el tipo y estado de los pavimentos deportivos, instrumentos deportivos y temperatura ambiente (Latorre et al., 2012; Ortín, Montero, Garcés de los Fayos y Olmedilla, 2010).

Muchos atletas asumen las lesiones como accidentes, es decir, el resultado de causas estables y globales que no pueden evitarse o controlarse, pero los investigadores han reconocido desde hace tiempo la inconsistencia de esas creencias y que el riesgo de sufrir una lesión deportiva es debido a una variedad de factores que se pueden prevenir, incluyendo las diferencias individuales de los atletas en personalidad y temperamento (Osborn, Blanton y Schwebel, 2009). Numerosos investigadores reconocen la importancia que los factores psicosociales desempeñan en la prevención de lesiones deportivas (Williams, 2001).

Determinados aspectos psicológicos y de la personalidad pueden establecer que un sujeto sea más propenso a adoptar conductas de riesgo y, por lo tanto, a accidentarse y lesionarse. Si bien, en un primer momento, los psicólogos centraron sus estudios en diferentes aspectos de la personalidad (Coddington y Troxel, 1980; Valliant, 1981), intentando establecer algún tipo de relación entre éstos y la probabilidad de lesionarse, los resultados, tal y como indican Rotella y Heyman (1991), no permiten establecer esta relación. Más recientemente han focalizado su atención en el análisis de aspectos más específicos de la psicología del deportista que se relacionan con el riesgo de lesión (Ali, Marivain, Hèas y Boulavais, 2006; Díaz et al., 2004; Olmedilla et al., 2006; Williams y Andersen, 1998).

A las personas con facilidad para lesionarse se les define como "personalidad propensa a la lesión», y se les atribuye una serie de características como agresividad, cólera, capacidad de atención, tendencia a sentirse ofendido y, en general, estado de ansiedad permanente (Pascual y Aragües, 1998). La propensión a los riesgos es entendida en términos de la variación individual en las medidas del riesgo. Sitkin y Pablo (1992) definen la propensión al riesgo como la toma de decisiones encaminada a evitar o tomar riesgos. Los factores psicológicos que más se han estudiado al respecto, se asocian con el estrés, la ansiedad competitiva, la impulsividad, las motivaciones de logro, el locus control, la búsqueda de sensaciones, la competencia percibida y autoconfianza, el autocontrol y los recursos de afrontamiento, la percepción del riesgo, el estado de ánimo y la concentración (Abenza, Olmedilla, Ortega y Esparza, 2009; Greening, Stoppelbein, Chandler y Elkin, 2005; Hansen, 2005; Ivarsson y Urban, 2010; Maddison y Prapavessis, 2007; Morrongiello y Rennie, 1998; Olmedilla, Ortega, Abenza y Boladeras, 2011; Williams y Andersen, 1998; Zuckerman, 1979).

La percepción del riesgo se incluye en muchos modelos de conducta saludable y es reconocido como un componente vital de la promoción de la salud (Van der Pligt, 1994). El marco conceptual para el estudio de la percepción del riesgo en las actividades deportivas se ha centrado en el paradigma psicométrico, introducido por Fischhoff, Slovic y Lichtenstein (1978). Desde este enfoque, se investiga mediante cuestionarios que incluyen un listado de diversas fuentes de peligro para que los sujetos las valoren, tanto en la dimensión cuantitativa del riesgo percibido en general, como en distintas características o atributos cualitativos del riesgo. Kontos (2004) destaca que la percepción del riesgo, la aceptación de éste y la estimación de la capacidad, representan importantes factores de riesgo psicológico para las lesiones en los deportes de los adolescentes. Además, los sujetos con altos niveles de toma de riesgos, bajos niveles de riesgo percibido y bajos niveles de estimación de habilidad, presentan más riesgo de lesiones. Horvath y Zuckerman (1993) han sugerido que las experiencias pasadas exitosas con la asunción de riesgos pueden llevar a una reducción del riesgo percibido, aumentando potencialmente la posibilidad de que un deportista corra riesgos en el deporte.

Por otro lado, la ansiedad competitiva puede ser un factor de predisposición a la lesión. Así, Olmedilla, Andreu, Ortín y Blas (2009) destacan la importancia de la ansiedad en la aparición de lesiones. En un estudio con futbolistas, Abenza, Olmedilla y Ortega (2010) señalan que los deportistas con menores niveles de autoconfianza y mayor ansiedad sufren lesiones graves y muy graves. Maddison y Prapavessis (2005) indican que la elevada ansiedad competitiva en una situación de estrés, provoca más riesgo de lesión. En este sentido, un mayor número (o gravedad) de lesiones se relaciona con puntuaciones más altas de ansiedad competitiva (Lavelle y Flint, 1996).

Otro factor que podría influir en el riesgo percibido y en la asunción de riesgos se relaciona con la autoeficacia y la competencia percibida. Bandura (1997) sugiere que los atletas que presentan una alta autoeficacia, son más propensos a intentar habilidades difíciles, por lo tanto, deben ser más propensos a tomar riesgos en el deporte, así, algunos individuos pueden sobreestimar su capacidad o percibirse a sí mismos mejores de lo que realmente son. Una sobreestimación de la capacidad puede dar lugar a una sensación de invencibilidad y a la decisión de involucrarse en conductas de mayor riesgo.

En este sentido, Kontos (2000) en un estudio realizado con una muestra de 253 futbolistas (chicos y chicas, entre 11 y 15 años), mostró que la sobreestimación de la capacidad se relacionaba positivamente con la asunción de riesgos, todo ello, unido a una baja percepción del riesgo de lesiones, pueden llevar a un deportista a ser imprudente en el deporte y aumentar su probabilidad de lesionarse.

Dentro de los factores de la personalidad, la búsqueda de sensaciones ha sido uno de los elementos de estudio más analizados en su relación con las conductas de riesgo y en 
este caso, con la accidentalidad y la percepción del riesgo. La búsqueda de sensaciones influye en la percepción del riesgo y es un claro predictor de los comportamientos arriesgados ( $\mathrm{Aj}$ cardi y Therme, 2008). En este sentido, la búsqueda de sensaciones ha sido ampliamente estudiada en la investigación de las diferencias individuales en la propensión a asumir riesgos en una amplia gama de comportamientos como la conducción de riesgo, comportamientos que contribuyen a las lesiones no intencionales, el consumo de alcohol, tabaco y drogas ilícitas, los malos hábitos alimenticios y la inactividad física, la práctica de deportes de riesgo, las conductas sexuales, la delincuencia u otras conductas antisociales y otros hábitos recreacionales (Bovard, 2008; Dunlop y Romer, 2010; Roberti, 2004; Zuckerman, 2007). Zuckerman (1979) considera que la búsqueda de sensaciones supone una necesidad de experimentar variadas y complejas sensaciones y el deseo de correr riesgos físicos y sociales, por el simple deseo de disfrutar de tales experiencias. El alto buscador de sensaciones se caracteriza por su tendencia a hacer aquellas cosas que un bajo buscador de sensaciones consideraría peligrosas y arriesgadas, la diferencia entre unos y otros es la distinta valoración que se hace del riesgo. Los buscadores de sensaciones asumen varios tipos de riesgos, entre ellos, asumen riesgos físicos en deportes peligrosos (Zuckerman, 1983). Las diferencias sexuales en la búsqueda de sensaciones parece que se pueden deber tanto a factores biológicos como a factores de socialización; en cuanto a la edad, parece existir una relación negativa entre edad y la búsqueda de sensaciones (Chico, 2000).

Otro factor de la personalidad que afecta a la percepción del riesgo es el egocentrismo de los adolescentes, entendido éste como la tendencia de los adolescentes a pensar que ellos son únicos, por lo que no pueden ser vulnerables a los riesgos para la salud como la persona media típica (Elkind, 1967). El egocentrismo en relación con la percepción del riesgo, refleja un sentimiento de inmortalidad, por tanto, este elemento se puede considerar como un factor de predisposición al accidente. En la medida en que percibimos que la probabilidad de que nos ocurra un hecho es menor que le ocurra a la persona media, esto no es sólo optimismo, sino que además no es realista (McKenna, 1993). Sin embargo, Greening et al. (2005) indican que un aumento del egocentrismo no predice los cambios en la percepción del riesgo. En este sentido, Dolcini et al. (1989) revelan una relación positiva pequeña entre el egocentrismo adolescente y el riesgo percibido de daño, siendo la relación significativa sólo para las niñas.

Los trabajos sobre evaluación de las conductas de riesgo de los deportistas resultan escasos, la mayoría de las investigaciones sobre la toma de decisiones en relación con el riesgo han utilizado las tareas de conveniencia, que exigen que el sujeto se imagine a sí mismo en diferentes contextos, con poca relación a situaciones de la vida real que suponen un riesgo de daño físico. Una de las críticas realizadas a este enfoque es la de haber trabajado con listas de riesgos seleccionadas de una forma bastante arbitraria (Cutter, 1993; Hendrickx, 1991) que incluyen riesgos representativos de ámbitos muy dispares. Por tanto, la selección de tareas es un aspecto importante a la hora de estudiar los riesgos (Morrongiello y Rennie, 1998). De los instrumentos encargados de valorar la propensión al riesgo, destacamos la escala de propensión al riesgo de Meertens y Lion (2008) que representa una medida sencilla de 7 ítems muy genéricos, que valora la tendencia individual a exponerse al riesgo. Y la prueba de tendencia al riesgo de Aguado, Rubio y Lucía (2011) basada en la ejecución comportamental ante una tarea. En la evaluación específica de la percepción del riesgo en el deporte, encontramos el estudio de Siesmaa, Blitvich, White y Finch (2011) en el que se desarrolla una encuesta para la medición de la participación de los niños en el deporte, la percepción del riesgo y la historia de lesiones. Latorre y Pantoja (2012) desarrollan un cuestionario de percepción del riesgo en la clase de Educación Física basándose en reactivos de imágenes relacionadas con instalaciones deportivas y juegos peligrosos.

Por tanto, la forma en que los sujetos se comportan en sus actividades deportivas en relación con la precisión a la hora de percibir y asumir los riesgos, de disponer de recursos de afrontamiento adecuados en la competición y la competencia motriz necesaria para desenvolverse eficazmente y de manera segura en el deporte, representan elementos muy interesantes en el estudio de la accidentalidad deportiva. Diversas investigaciones han intentado identificar los factores relacionados con el incremento del número de lesiones en la práctica deportiva. La identificación de estos factores podría capacitar a entrenadores y deportistas para modificar los programas de entrenamiento y así prevenir futuras lesiones (Fernández, de la Cruz, Cueto, Salazar y de la Cruz Campos, 2008). Todo ello, hace necesario la existencia de instrumentos que permitan conocer el valor de aquellos factores que pueden predisponer a un sujeto al accidente deportivo. La escasez de este tipo de instrumentos específicos para el deporte, motivó la creación de la escala de propensión al accidente deportivo (PAD-22).

El objetivo de este estudio es desarrollar un instrumento que permita identificar a los deportistas que presentan mayor propensión al accidente y a la lesión deportiva, analizando la influencia del tipo de deporte practicado y del sexo.

\section{Método}

\section{Participantes}

La muestra de este estudio está compuesta por 118 sujetos (61\% hombres y 39\% mujeres), de edades comprendidas entre 18 y 38 ańos (22.42 \pm 4.01 años), divididos en 3 grupos. El primer grupo está integrado por 35 sujetos (32 hombres y 3 mujeres) que practican rugby federado de forma regular; el 
segundo grupo está compuesto por 35 sujetos (7 hombres y 28 mujeres) que practican voleibol federado de forma regular; y el tercer grupo está formado por 48 sujetos (33 hombres y 15 mujeres) que practican deporte de forma recreativa, entendiendo deporte recreativo como aquél que se realiza sin estar federado y sin competiciones oficiales. En este caso, los sujetos practican aerobic, ciclismo de montaña, carrera pedestre, tonificación muscular con pesas y pádel. Se trata de una muestra no probabilística por conveniencia. Los deportistas que participan en el estudio, tanto los de rugby como los de voleibol, pertenecen a varios clubes de las provincias de Córdoba y Jaén, mientras que los sujetos que realizan deporte de forma recreativa, son alumnos de Magisterio de Educación Física de la Universidad de Córdoba. Otras características específicas de los sujetos se muestran en la tabla 1 .

\section{Instrumentos}

Para la realización del presente estudio se han analizado dos constructos psicológicos: la búsqueda de sensaciones y la propensión al accidente y lesión deportiva. Asimismo, se han tenido en cuenta otra serie de datos para contextualizar y definir la muestra, como son la edad, el sexo, el nivel de estudios, si se habían lesionado en ese año (Sí o No) y el número de años de práctica deportiva. Para medir el constructo "búsqueda de sensaciones" se ha utilizado la Escala de Búsqueda de Sensaciones Forma V (Sensation Seeking Scale, SSS-V) de Zuckerman, Eysenck y Eysenck (1978), en la versión española (Pérez y Torrubia, 1986). Se trata de un cuestionario autoadministrado, formado por 40 preguntas dicotómicas (Sí-No). La escala está subdividida en 4 dimensiones de 10 ítems cada una: Búsqueda de emociones y aventuras (BEA), Búsqueda de experiencias (BEX), Desinhibición (DES) y Susceptibilidad al aburrimiento (SAB). Cada dimensión da lugar a una puntuación de 0 (la más baja) a 10 (la más alta), existiendo además una puntuación total que sería la suma de las puntuaciones obtenidas en las cuatro dimensiones (de 0 a 40). Pérez y Torrubia (1986) indican coeficientes de fiabilidad de .94 para BEA, .68 para BEX, .92 para DES , .87 para SAB y .90 para el total de la escala.

La escala PAD-22, es un cuestionario de autoinforme compuesto por 22 ítems que describen unas variables que condicionan la posibilidad de sufrir un accidente deportivo. En el protocolo del instrumento se solicita que se completen las cuestiones expresadas en éste, de acuerdo a una escala tipo Likert (1, significa muy en desacuerdo y 6 , muy de acuerdo). El diseño y elaboración de la escala se desarrolló en tres fases. En una primera, se realizó una revisión bibliográfica de aquellos factores que condicionan la accidentalidad en el deporte relacionados con el propio sujeto. Basándonos en estos antecedentes se empezó a elaborar una serie de cuestiones referenciadas a los factores psicológicos y de personalidad que determinan la accidentalidad, todo ello, adaptado al ámbito de la actividad físico-deportiva. Además, se emplearon como referencia, variables de la escala de búsqueda de sensaciones de Pérez, Ortet, Plá y Simó (1987) y del cuestionario de autoconcepto físico "CAF" de Goñi, Ruiz y Liberal (2004), configurándose un cuestionario inicial de 30 ítems. En una segunda fase, y como elemento de validez de contenido, se seleccionó a un grupo de seis expertos de Educación Física, con experiencia investigadora en el ámbito de la accidentalidad deportiva; solicitándoles un juicio crítico sobre los elementos iniciales de la escala y posibles aportaciones de ítems nuevos, lo cual condujo a la eliminación de 5 ítems basándonos en su contenido, inteligibilidad y en los que los jueces presentaban discrepancias en la valoración (prueba de concordancia de Kendall). Quedando una escala preliminar de 25 ítems. En una tercera fase, objeto de este estudio, se procedió a la validación factorial, análisis de fiabilidad y validez concurrente del instrumento con una muestra de 118 deportistas. La personalidad de los individuos, el temperamento y la búsqueda de sensaciones en particular, también se correlaciona con el riesgo de lesión en los deportes (Osborn et al., 2009), en este sentido, se realizó una validez concurrente con el cuestionario SSS-V en consonancia con estudios previos (Aguado et al., 2011).

\section{Procedimiento}

Previamente a la administración de los cuestionarios se completó por parte de los sujetos un formulario de consentimiento informado, en donde se destaca la confidencialidad de los cuestionarios, así como el carácter voluntario de su participación. De manera autoadministrada se llevó a cabo el cuestionario PAD-22 por pequeńos grupos y en presencia de un investigador del estudio. Seguidamente, los sujetos completaron la escala SSS-V. Se atendieron las dudas surgidas y se garantizó la confidencialidad y el anonimato de las respuestas. La duración de aplicación de los cuestionarios fue de aproximadamente 40 minutos.

\section{Análisis estadístico}

Los datos de este estudio se han hallado mediante el programa estadístico SPSS., v.18.0 para Windows, (SPSS Inc, Chicago, USA). Se ha empleado la prueba Chi cuadrado y U de Mann-Whitney para analizar la homogeneidad de grupos en relación a las variables descriptoras de la muestra. Se han calculado los análisis de fiabilidad interna (Cronbach's alpha) de los cuestionarios. Además, se ha utilizado la estadística descriptiva (medias, DT), análisis factorial (Varimax con Kaiser), correlaciones (Pearson) y diferencias de medias (ANCOVA y Prueba t), empleando como covariable el sexo en la comparación de los grupos de deportistas. El nivel de significación fue de $\mathrm{p}<.05$. 


\section{Resultados}

\section{Características de la muestra}

En la tabla 1 se muestran las características de los sujetos. Se destaca que en el rugby existe una mayor presencia de varo- nes, circunstancia que se invierte en el voleibol donde encontramos mayor porcentaje de mujeres.

Tabla 1. Características de la muestra.

\begin{tabular}{|c|c|c|c|c|c|}
\hline & & $\begin{array}{c}\text { Rugby } \\
\mathrm{n}=35\end{array}$ & $\begin{array}{c}\text { Voleibol } \\
\mathrm{n}=35\end{array}$ & $\begin{array}{c}\begin{array}{c}\text { Deporte recreativo } \\
\mathrm{n}=48\end{array} \\
\end{array}$ & $\mathrm{p}$ \\
\hline \multirow{2}{*}{$\begin{array}{l}\text { Tiene lesiones } \\
\mathrm{n}(\%)^{*}\end{array}$} & Sí & $13(37.1)$ & $11(32.4)$ & $25(52.1)$ & \multirow{2}{*}{ NS } \\
\hline & No & $22(62.9)$ & $23(67.6)$ & $23(47.9)$ & \\
\hline \multirow[t]{2}{*}{ Estudios n (\%) } & Secundaria & $14(40.0)$ & $21(60.0)$ & $0(0.0)$ & \multirow{2}{*}{.000} \\
\hline & Universitario & $21(60.0)$ & $14(40.0)$ & $48(100.0)$ & \\
\hline \multicolumn{2}{|c|}{ Años de práctica. Media (DT) } & $5.70(5.97)$ & $10.39(4.49)$ & $14.19(4.58)$ & .000 \\
\hline \multicolumn{2}{|c|}{ Edad (años). Media (DT) } & $22.11(4.14)$ & $21.89(3.89)$ & $23.02(4.00)$ & NS \\
\hline \multirow{2}{*}{ Sexo n (\%) } & Hombre & $32(91.4)$ & $7(20)$ & $33(68.8)$ & \multirow{2}{*}{.000} \\
\hline & Mujer & $3(8.6)$ & $28(80)$ & $15(31.3)$ & \\
\hline
\end{tabular}

*un valor perdido en voleibol. NS. No significativo.

\section{Validez factorial}

Una vez confirmado que el tamaño muestral era adecuado para estudiar la calidad técnica del instrumento ya que el número de sujetos recomendado debe ser entre dos y diez veces el número de ítems (Pallás y Villa, 2004), se analizaron las evidencias de validez factorial de la escala. El objetivo principal es comprobar si los 25 ítems de la escala se pueden resumir de alguna forma, es decir, si existen rasgos comunes entre ellos. Paso previo al análisis factorial, se calculó la homogeneidad del cuestionario, lo que nos permitió eliminar 3 ítems que presentaban un bajo nivel de discriminación $(<200$, correlación elemento-total corregida, tabla 3), de acuerdo con las recomendaciones de Elbel (1965). Quedándose constituida la escala con 22 ítems. Seguidamente, se recurre al análisis factorial por el método de los componentes principales, como forma de agrupar estos reactivos en factores o componentes que puedan explicar la varianza observada en las respuestas ofrecidas por los sujetos. A continuación, analizamos el grado de correlación entre las variables, que ha de ser alto para poder realizar el análisis factorial. El índice de adecuación muestral KMO alcanza un valor de 0.734 y la prueba de esfericidad de Bartlett es de 969.989 ( $\mathrm{p}<.00$ ). Estos datos hacen que se rechace la hipótesis nula de que la matriz de correlación inter-ítems es identidad y se considera que las respuestas están sustancialmente relacionadas, lo que justifica la realización del análisis factorial. A continuación, determinamos las comunalidades o proporción de varianza que es explicada por los factores comunes. En general, al no existir valores próximos a cero, se puede afirmar que los 22 ítems son explicados por los componentes. El análisis de los componentes principales y Rotación Varimax puso de manifiesto, tras nueve iteraciones, la convergencia en cinco factores que explican el $61.68 \%$ de la varianza. Factor 1, definido como búsqueda de sensaciones (BS), factor 2, como asunción de riesgos (AR), factor 3, como competencia percibida (CP), factor 4, como percepción del riesgo (PR) y el factor 5 como competitividad (COMP). El primer componente es el que más cantidad de varianza explica (24.36\%), y va decreciendo hasta llegar al último factor que sólo lo hace en un $6.20 \%$. Los ítems quedaron configurados de la forma como se puede apreciar en la tabla 2, ordenados de acuerdo al grado de saturación.

Tabla 2. Matriz de componentes rotados y coeficiente alfa de Cronbach por factor.

\begin{tabular}{lc}
\hline Ítems & \multicolumn{2}{c}{ Componentes } \\
\hline Me gusta tirarme desde el trampolín más alto de la piscina. & Factor 1 Factor 2 Factor 3 Factor 4 Factor 5 \\
Me gusta montarme en una montańa rusa. & .801 \\
Me gusta explorar un bosque desconocido haciendo senderismo o en bicicleta, aunque & .736 \\
pueda perderme. & .727 \\
Algunas veces me gusta hacer cosas que impliquen pequeños sobresaltos. & .715
\end{tabular}


Siempre soy el primero en ofrecerme para hacer las actividades más arriesgadas. $\quad .713$

Me gusta hacer acampada en lugares deshabitados y alejados.

No me importa patinar sin casco ni protecciones.

.866

.736

.682

Suelo soltarme de manos al conducir la bicicleta.

.638

Hago deporte estando lesionado.

Mi cuerpo no me transmite sensaciones positivas.

Frecuentemente estoy mal por alguna cuestión relacionada con mi apariencia física.

Creo que, comparado con la mayoría, mi cuerpo no parece estar en la mejor forma física.

Hacer una voltereta en el aire es poco peligroso.

Saltar el potro es poco peligroso.

Como nado muy bien, hacer piragüismo sin chaleco salvavidas no supone ningún riesgo para mí.

Cuando hago deporte, si no gano, no me divierto.

Necesito superarme para ser el mejor.

Cuando pierdo me siento muy mal conmigo mismo.

En el ítem 1 (Anexo) debe invertirse su valoración. En los factores 3 y 4, a mayor puntuación del factor peor competencia percibida y menor percepción del riesgo respectivamente.

\section{Análisis de fiabilidad}

Una vez definida la validez de la escala, calculamos la fiabilidad de la misma mediante el coeficiente alfa de Cronbach, que alcanza un valor de .82 en el total de la escala, .84 en BS,
.75 en AR, .69 en CP, .74 en PR y .63 en COMP, lo que indica una adecuada consistencia interna del instrumento, o lo que es lo mismo, que es confiable. En la tabla 3 se muestra la fiabilidad de la escala mediante la correlación ítem-total, o lo que es lo mismo, el contraste de medias entre los grupos con puntuaciones totales más altas y más bajas. En nuestro caso, oscilan entre .80 y .83 , una franja muy pequeña que garantiza la unidimensionalidad básica, dado que se pretende medir un rasgo único como es la propensión al accidente deportivo.

Tabla 3. Correlación de los ítems con el total de la escala.

\begin{tabular}{lcccc}
\hline & $\begin{array}{c}\text { Media de la escala si se } \\
\text { elimina el elemento }\end{array}$ & $\begin{array}{c}\text { Varianza de la escala si } \\
\text { se elimina el elemento }\end{array}$ & $\begin{array}{c}\text { Correlación elemento- } \\
\text { total corregida }\end{array}$ & $\begin{array}{c}\text { Alfa de Cronbach si se } \\
\text { elimina el elemento }\end{array}$ \\
\hline Ítem 1 & 71.47 & 264.70 & .53 & .80 \\
Ítem 2 & 70.76 & 277.38 & .39 & .81 \\
Ítem 3 & 71.07 & 286.61 & .22 & .82 \\
Ítem 4 & 70.87 & 271.33 & .54 & .81 \\
Ítem 5 & 71.59 & 263.53 & .65 & .80 \\
Ítem 6 & 71.41 & 272.91 & .49 & .81 \\
Ítem 7 & 71.92 & 276.34 & .34 & .82 \\
Ítem 8 & 71.46 & 279.73 & .29 & .82 \\
Ítem 9 & 72.15 & 266.12 & .52 & .81 \\
Ítem 10 & 72.02 & 272.40 & .51 & .81 \\
Ítem 11 & 72.71 & 314.55 & -.29 & .83 \\
Ítem 12 & 72.52 & 285.18 & .32 & .82 \\
Ítem 13 & 72.68 & 289.74 & .24 & .82 \\
Ítem 14 & 72.17 & 290.33 & .21 & .82 \\
Ítem 15 & 72.34 & 279.44 & .38 & .81 \\
Ítem 16 & 71.24 & 275.03 & .46 & .81
\end{tabular}




\begin{tabular}{lllll} 
Ítem 17 & 71.33 & 267.57 & .59 & .80 \\
Ítem 18 & 72.95 & 279.89 & .41 & .81 \\
Ítem 19 & 72.72 & 284.58 & .32 & .82 \\
Ítem 20 & 71.47 & 285.58 & .30 & .82 \\
Ítem 21 & 71.35 & 278.57 & .39 & .81 \\
Ítem 22 & 71.17 & 289.28 & .28 & .82 \\
\hline
\end{tabular}

\section{Validez concurrente}

Para comprobar la validez concurrente del cuestionario PAD22 procedimos a realizar un análisis de correlación Pearson con la Escala SSS-V. Existe una alta correlación entre las dimensiones de la escala PAD-22 y una correlación aceptable con el resto de dimensiones y el total de puntuación de la escala SSS-V (Tabla 4).

Tabla 4. Correlación Pearson entre el cuestionario PAD-22 y SSS-V.

\begin{tabular}{|c|c|c|c|c|c|c|c|c|c|c|}
\hline & BS & $\mathrm{AR}$ & $\mathrm{CP}$ & $\mathrm{PR}$ & COMP & BEA & BEX & DES & SAB & Total SSS-V \\
\hline BS & 1 & $.24^{* *}$ & & $.41^{* *}$ & $.25^{* *}$ & $.45^{* *}$ & $.24^{* *}$ & & & $.43^{* *}$ \\
\hline AR & & 1 & & $.28^{* *}$ & $.38^{* *}$ & & & $.22^{*}$ & & $.19^{*}$ \\
\hline $\mathrm{CP}$ & & & 1 & $.23^{*}$ & & & & & & \\
\hline PR & & & & 1 & $.29^{* *}$ & $.19^{*}$ & & $.20^{*}$ & & \\
\hline COMP & & & & & 1 & & & & & \\
\hline
\end{tabular}

Relación entre la propensión al accidente, búsqueda de sensaciones, tipo de deporte y sexo

En la tabla 5 se muestran los resultados de la comparación por grupos de deportistas en la escala PAD-22 y SSS-V. Se observan diferencias significativas $(\mathrm{p}<.05)$ en las dimensiones de PR y COMP de la escala PAD-22 y en la BEA y en el total de la escala SSS-V. En las comparaciones por pares mediante ajuste Bonferroni, se encuentran diferencias significativas $(p<.05)$ entre el rugby y el deporte recreativo en la dimensión de PR, presentando por tanto los jugadores de rugby menor percepción del riesgo. En la dimensión COMP, en el rugby en relación con el deporte recreativo se presentan mayores valores de competitividad ( $\mathrm{p}<.01$ ), igualmente en el voleibol con respecto al deporte recreativo $(\mathrm{p}<.01)$. En la dimensión BEA, el rugby presenta mayores valores que el voleibol $(p<.05)$. Por último, en el total de la escala SSS-V, el rugby presenta mayor puntuación que el voleibol ( $\mathrm{p}<.05)$.

Tabla 5. Escalas PAD-22 y SSS-V por deporte practicado.

\begin{tabular}{llccccccc}
\hline Escala & Dimensión & $\begin{array}{c}\text { Rugby } \\
(\mathrm{n}=35) \\
\text { Media (DT) }\end{array}$ & $\begin{array}{c}\text { Voleibol } \\
(\mathrm{n}=35) \\
\text { Media (DT) }\end{array}$ & $\begin{array}{c}\text { Deporte recreativo } \\
(\mathrm{n}=48) \\
\text { Media (DT) }\end{array}$ & F & p & $\begin{array}{c}\text { Eta } \\
\text { cuadrado }\end{array}$ & $\begin{array}{c}\text { Potencia } \\
\text { observada }\end{array}$ \\
\hline BS & $27.46(6.43)$ & $22.20(7.84)$ & $22.52(8.90)$ & 2.375 & NS & 0.04 & 0.47 \\
PAD-22 & AR & $14.23(6.33)$ & $14.03(5.65)$ & $12.08(5.66)$ & 1.925 & NS & 0.03 & 0.39 \\
& PR & $11.14(3.56)$ & $10.40(5.15)$ & $11.20(4.02)$ & 0.345 & NS & 0.00 & 0.10 \\
& $15.31(3.99)$ & $13.00(5.38)$ & $11.91(5.13)$ & 4.198 & .01 & 0.07 & 0.72 \\
& COMP & $15.89(3.66)$ & $14.90(4.23)$ & $12.09(4.05)$ & 11.78 & .00 & 0.17 & 0.99 \\
SSS-V & $6.94(2.39)$ & $4.71(2.90)$ & $6.13(2.55)$ & 3.213 & .04 & 0.05 & 0.60 \\
& BEA & $5.53(1.84)$ & $5.75(1.43)$ & $5.65(1.95)$ & 0.119 & NS & 0.00 & 0.06 \\
& DEX & $6.17(2.00)$ & $4.33(1.99)$ & $5.30(2.28)$ & 2.852 & NS & 0.04 & 0.54 \\
& SAB & $4.21(2.04)$ & $3.57(2.18)$ & $4.43(2.58)$ & 0.808 & NS & 0.01 & 0.18 \\
Datos expresados en media y desviación típica (DT). Búsqueda de sensaciones (BS). Asunción de riesgos (AR). Competencia percibida (CP). \\
Percepción del riesgo (PR). Competitividad (COMP). Búsqueda de emociones y aventura (BEA). Búsqueda de experiencias (BEX). Desinhibi- \\
ción (DES). Susceptibilidad al aburrimiento (SAB). Sensation Seeking Scale (SSS-V). NS. No significativo. & &
\end{tabular}


En la tabla 6 se exponen los resultados de las escalas PAD-22 y SSS-V según el sexo. Los varones muestran mayor valoración que las mujeres, con diferencias significativas $(\mathrm{p}<.05)$, en

Tabla 6. Escalas PAD-22 y SSS-V según el sexo.

\begin{tabular}{|c|c|c|c|c|}
\hline Escala & Dimensión & $\begin{array}{c}\text { Hombres } \\
(\mathrm{n}=72) \\
\text { Media (DT) }\end{array}$ & $\begin{array}{c}\text { Mujeres } \\
(\mathrm{n}=46) \\
\text { Media (DT) }\end{array}$ & $\mathrm{p}$ \\
\hline \multirow{5}{*}{ PAD-22 } & BS & $26.01(7.17)$ & $20.76(8.63)$ & .00 \\
\hline & $\mathrm{AR}$ & $13.53(6.02)$ & $12.89(5.76)$ & NS \\
\hline & $\mathrm{CP}$ & $11.06(4.14)$ & $10.76(4.45)$ & NS \\
\hline & PR & $13.76(4.95)$ & $12.42(5.18)$ & NS \\
\hline & COMP & $14.79(4.17)$ & $12.71(4.25)$ & .01 \\
\hline \multirow{5}{*}{ SSS- V } & BEA & $6.45(2.34)$ & $5.15(3.13)$ & .01 \\
\hline & BEX & $5.44(1.79)$ & $5.95(1.72)$ & NS \\
\hline & DES & $5.74(2.16)$ & $4.60(2.14)$ & .00 \\
\hline & SAB & $4.32(2.47)$ & $3.78(2.06)$ & NS \\
\hline & Total SSS-V & $21.89(5.94)$ & $19.50(6.01)$ & .04 \\
\hline \multicolumn{5}{|c|}{$\begin{array}{l}\text { Datos expresados en media y desviación típica (DT). Búsqueda de sensacio- } \\
\text { nes (BS). Asunción de riesgos (AR). Competencia percibida (CP). Percepción } \\
\text { del riesgo (PR). Competitividad (COMP). Búsqueda de emociones y } \\
\text { aventura (BEA). Búsqueda de experiencias (BEX). Desinhibición (DES). } \\
\text { Susceptibilidad al aburrimiento (SAB). Sensation Seeking Scale (SSS-V). NS. } \\
\text { No significativo. }\end{array}$} \\
\hline
\end{tabular}

\section{Discusión}

El objetivo de este estudio ha sido elaborar un instrumento de propensión al accidente deportivo en deportistas. El cuestionario PAD-22 constituye un instrumento inédito y original que ha mostrado fiabilidad y adecuada validez de contenido y factorial. En cuanto a la fiabilidad, la escala ha mostrado buena consistencia interna con un coeficiente alfa de Cronbach igual a .82. Las saturaciones de cada uno de los ítems con sus respectivos factores son altas. Por otro lado, las correlaciones entre factores indican cierta relación y dependencia entre ellos. Podemos indicar además que se produce una aceptable validez concurrente del cuestionario PAD-22 con el SSS-V y los resultados confirman los hallazgos encontrados en la literatura que vienen a corroborar el hecho de que pueda existir algún tipo de relación teórica y conceptual entre los factores de propensión al accidente y la búsqueda de sensaciones que predispone a los deportistas a conductas de riesgo (Osborn et al., 2009). Por todo ello, podemos afirmar que los datos confirman la validez del cuestionario, con una estructura de cinco factores.

Zuckerman (1983) define los deportes de alto riesgo como aquéllos donde existen evidencias de accidentes graves, como por ejemplo: el paracaidismo, las carreras de coches, el vuelo sin motor, el buceo libre y el esquí alpino. Por otro lado, las dimensiones BS y COMP de la escala PAD-22 y en las dimensiones de BEA, DES y en el total de la escala SSS-V.

dentro de los deportes menos peligrosos encontraríamos el golf, la natación o el maratón, donde se encuentran amenazas para la salud y lesiones, pero el riesgo de accidentes graves es muy bajo. Entre estos dos extremos, se incluyen deportes de moderado riesgo físico como el fútbol americano o el rugby, donde las lesiones graves son factibles, pero el riesgo de muerte es relativamente bajo. La investigación sobre las relaciones entre la búsqueda de sensaciones y la práctica deportiva se han centrado en definir las diferencias entre las personas que se involucran en los deportes extremos y los que prefieren formas menos arriesgadas de deporte o actividad física de recreación. En este estudio, teniendo en cuenta que el rugby es considerado como un deporte de riesgo moderado, en comparación con el voleibol y el deporte recreativo que conllevan un riesgo bajo, hemos encontrado mayores niveles de competitividad, menor percepción del riesgo, mayor búsqueda de emociones y aventuras y mayor puntuación en la escala total SSS-V en el rugby que en estos últimos. Estos resultados están en consonancia con otros trabajos en los que se comparan deportes con alto riesgo físico con deportes menos peligrosos (Diehm y Armatas, 2004; Franques et al., 2003; Guszkowska y Botdak, 2010; Jack y Ronan, 1998) o deportes de contacto y no contacto (O' Sullivan, Zuckerman y Kraft, 1998). En un estudio similar, Potgieter y Bisschoff (1990) compararon la escala de búsqueda de sensaciones en 35 jugadores de rugby y 32 maratonianos; los jugadores de rugby obtuvieron puntuaciones más altas en la escala de búsqueda de sensaciones y en la dimensión búsqueda de emoción y aventura. Rhea y Martin (2010) indican que los atletas de deportes alternativos de riesgo (moto-cross, esquí acuático) son más reservados, auto-suficientes y presentan mayor búsqueda de sensaciones que los atletas de deportes tradicionales (tenis y voleibol). De manera semejante, los triatletas en comparación con los deportistas de parapente, puenting y paracaidismo tenían mayores niveles de percepción personal del riesgo. Se observó una relación negativa entre la vulnerabilidad a una lesión grave y la percepción personal del riesgo entre todos los participantes. Este resultado apoya la hipótesis de la asociación entre la percepción del riesgo y la auto-eficacia que se ha observado entre los deportistas (Martha y Laurendeau, 2010).

En relación con las diferencias entre sexos, independientemente de la participación deportiva, en este estudio los varones obtienen mayor puntuación en las dimensiones de búsqueda de sensaciones y competitividad del PAD-22 y de búsqueda de emociones y aventura, desinhibición y el total de la escala de SSS-V. En este sentido, Schroth (1995) indica que los dos grupos de hombres (deportistas y no deportistas) 
obtienen valores de búsqueda de sensaciones superiores a las mujeres; además, los deportistas, tanto hombres como mujeres, puntúan más alto en la búsqueda de sensaciones que los no deportistas. Hartman y Rawson (1992) destacan que los varones puntúan más alto que las mujeres en búsqueda de sensaciones, independientemente de su participación deportiva y que los deportistas obtienen calificaciones más altas que los no deportistas. Franken, Hill y Kierstead (1994) indican que las mujeres en comparación con los hombres se interesan más en la gimnasia y el patinaje artístico, mientras que los varones por el hockey, fútbol, baloncesto, golf, tenis y boxeo, en consecuencia, los varones obtienen puntuaciones más altas en búsqueda de sensaciones y competitividad.

\section{Limitaciones del estudio y perspectivas de futuro}

Se presenta una versión preliminar del cuestionario PAD-22, que podrá ser mejorada en sucesivas aplicaciones contando con un mayor número de participantes y un seguimiento preciso de la accidentalidad y lesiones de los mismos en cada temporada deportiva. Todo ello, requiere que los datos aquí aportados con esta muestra piloto deberían ser depurados y ampliados posteriormente en una muestra más numerosa y con una representación de otros deportes, además de los ya contemplados en el presente estudio. Además, habría que establecer la relación entre el número y gravedad de lesiones de los deportistas con las dimensiones de la escala PAD-22.

\section{Conclusiones}

La principal conclusión es que los análisis psicométricos del presente estudio confirman la validez y fiabilidad de la versión inicial del PAD-22. La escala puede servir como instrumento válido para ser utilizado por entrenadores y técnicos deportivos a la hora de detectar a deportistas con elevada búsqueda de sensaciones, alta asunción de riesgos, elevada competencia percibida, reducida percepción del riesgo y elevada competitividad, en relación con las características de la acción deportiva y los requisitos de la tarea motriz. Todo ello, permitiría elaborar estrategias conductuales y contextuales necesarias para prevenir lesiones y accidentes deportivos, permitiendo a su vez que el diseño de actividades físico-deportivas se adecúe al nivel de autoeficiencia, personalidad y percepción del riesgo del deportista en cuestión.

\section{Referencias}

1. Abenza, L., Olmedilla, A., Ortega, E. y Esparza, F. (2009). Estados de ánimo y adherencia a la rehabilitación de deportistas lesionados. Apunts Medicina de l'Esport, 161 (44), 29-37.

2. Abenza, L., Olmedilla., A. y Ortega, E. (2010). Effect of injuries on psychological variables among under-19 soccer players. Revista Latinoamericana de Psicolgía, 42 (2), 265-277.

3. Aguado, Rubio V.J y Lucía, B. (2011). La Prueba de Tendencia al Riesgo (PTR): Una propuesta para un test informatizado de ejecución conductual para evaluar la tendencia al riesgo. Anales de psicología, 27 (3), 862-870.

4. Ajcardi, R. y Therme, P. (2008). Sensation seeking, affective profile, and sports behavior in the prediction of risk. International Journal of Sport Psychology, 39 (4), 356-372.

5. Ali, M., Mariavain, T., Hèas, A. y Boulavais, H. (2006). Analysis of coping strategies used by players of tennis men and women toward a severe athletic injury. Annales Medico Psychologiques, 10, 1016.

6. Andersen, M. B. y Williams, J. M. (1988). A model of stress and athletic injury: Prediction and prevention. Journal of Sport and Exercise Psychology, 10 (3), 294-306.

7. Bandura, A. (1997). Self-efficacy: The exercise of control. New York: Freeman.

8. Bovard, R. S. (2008). Risk behaviors in high school and college sport. Curr Sports Med Rep, 7(6), 359-366.

9. Chico, L. (2000). Búsqueda de sensaciones. Psicothema, 12 (2), 229-235.

10. Coddington, R. D. y Troxel, J. R. (1980). The effects of emotional factors on football injury rates, a pilot study. Journal of Human Stress, 6 (4), 3-5.

11. Cutter, S.L. (1993). Living with Risk. Londres: Edward Arnold.

12. Díaz, P., Buceta, J. M. y Bueno, A. M. (2004). Situaciones estresantes y vulnerabilidad a las lesiones deportivas: un estudio con deportistas de equipo. Revista de Psicología del Deporte, 14 (1), 7-24.

13. Diehm, R. y Armatas C. (2004). Surfing: an avenue for socially acceptable risk-taking, satisfying needs for sensation seeking and experience seeking. Personality and Individual Differences, 36 (3), 663-677.

14. Dolcini, M. M., Cohn, L. D., Adler, N. E., Millstein, S. G., Irwin, C.

E., Kegeles, S. M., et al. (1989). Adolescent egocentrism and feelings of invulnerability: Are they related? Journal of Early Adolescence, 9 (4), 409-418.

15. Dunlop, S. M., y Romer, D. (2010). Adolescent and young adult crash risk: sensation seeking, substance use propensity and substance use behaviors. J Adolesc Health, 46 (1), 90-92.

16. Elbel, R. L. (1965). Measuring educational achievement. Englewoods Cliffs, N.J: Prentice-Hall.

17. Elkind, D. (1967). Egocentrism in adolescence. Child Development, 38 (4), 1025-1034.

18. Fernández, A., de la Cruz, J.C., Cueto, B., Salazar, S. y de la Cruz Campos, J.C. (2008). Predicción de lesiones deportivas mediante modelos matemáticos. Apunts. Medicina de l'Esport, 43 (157), 41-4.

19. Fischhoff, B., Slovic, P. y Lichtenstein, S. (1978). Fault trees: Sensitivity of estimated failure probabilities to problem representation. Journal of Experimental. Psychology: Human Perception and Performance, 4 (2), 330-344.

20. Franken, R., Hill, R. y Kierstead, J. (1994). Sport interest as predicted by the personality measures of competitiveness, mastery, instrumentality, expressivity, and sensation seeking. Personality and Individual Differences, 17 (4), 467-476.

21. Franques, P., Auriacombe, M., Piquemal, E., Verger, M., Brisseau-Giménez, S., Grabot, D. y Tignol, J. (2003). Sensation seeking as a common factor in opioid dependent subjects and high risk sport practicing subjects. A cross sectional study. Drug and Alcohol Dependence, 69 (2), 121-126.

22. Goñi, A., Ruiz, S. y Liberal, I. (2004). Propiedades psicométricas de un nuevo cuestionario para la medida del autoconcepto físico. Revista de Psicología del Deporte, 13 (2), 195-21.

23. Greening, L., Stoppelbein, L., Chandler, C. y Elkin, D. (2005). Predictors of Children's and Adolescents' Risk Perception. Journal of Pediatric Psychology, 30 (5), 425-435.

24. Guszkowska, M. y Botdak, A. (2010). Sensation Seeking in Males Invol- 
ved in Recreational High Risk Sports. Biology of Sport, 27(3), 157-162.

25. Hansen, C.P. (2005). Personality characteristics of the accident involved employee. Journal of Business y Psychology, 2 (4), 346-365.

26. Hartman, M. y Rawson, H. (1992). Differences in and correlates of sensation seeking in male and female athletes and nonathletes. Personality and Individual Differences, 13 (7), 805-812.

27. Hendrickx, L.C. (1991). How Versus How Often. The role of Scenario Information and Frequency Information in Risk Judgment and Risky Decision Making. Groningen: Rijksuniversiteit Groningen.

28. Horvath, P. y Zuckerman, M. (1993). Sensation seeking and risk appraisal and risk behavior. Personality \& Individual Differences, 14 (1), 41-52.

29. Ivarsson, A. y Urban, J. (2010). Psychological factors as predictors of injuries among senior soccer players. A prospective study. Journal of Sports Science and Medicine, 9, 347-352.

30. Jack, S. J. y Ronan, K. R. (1998). Sensation seeking among high and low risk sports participants. Personality and Individual Differences, 25 (6), 1063-1083

31. Kontos, A. P. (2000). The effects of perceived risk, risktaking behaviors, and body size on injury in youth sport. Eugene, OR: Microform Publications, University of Oregon.

32. Kontos, A. P. (2004). Perceived risk, risk taking, estimation of ability and injury among adolescent sport participants. Journal of Pediatric Psychology, 29 (6), 447-455.

33. Latorre, P.A., Mejía, J.A., Gallego, M., Muñoz, A., Santos, M. y Adell, M. (2012). Análisis de la seguridad de las instalaciones deportivas de las sedes de los juegos deportivos provinciales de Jaén. Journal of Sport and Health Research, 4 (1), 57-66.

34. Latorre, P.A., y Pantoja, A. (2012). Diseńo y validación de una escala de percepción del riesgo en actividades físico-deportivas escolares. Retos 21 (1), 25-29.

35. Lavelle, L. y Flint, F.A. (1996). The relationship of stress, competitive anxiety, mood state, and social support to athletic injury. Journal of Athletic Training, 31 (4), 296-299.

36. Maddison, R. y Prapavessis, H. (2007). Preventing sport injuries: A case for psychology intervention. In: Psychological bases of sport injuries (pp.2538). Ed: Pargman, D. Morgantown, WV: Fitness Information Technology.

37. Martha, C. y Laurendeau, J. (2010). Are perceived comparative risks realistic among high-risk sports participants?. International Journal of Sport and Exercise Psychology, 8 (2), 129-14.

38. McKenna, F.P. (1993). It won't happen to me: Unrealistic optimism or illusion of control? Bristish Journal of Psychology, 84 (1), 39-50.

39. Meertens, M.R. y Lion, R. (2008). Measuring an individual's tendency to take risks: The risk propensity scale. Journal of Applied Social Psycho$\log y, 38(6), 1520-1526$.

40. Morrongiello, B. A. y Rennie, H. (1998). Why do boys engage in more risk taking than girls? The role of attributions, beliefs, and risk appraisals. Journal of Pediatric Psychology, 23 (1), 33-43.

41. Olmedilla, A., Andreu, M.D., Ortín, F.J. y Blas, A. (2009). Ansiedad competitiva, percepción de éxito y lesiones: un estudio en futbolistas. Revista Internacional de Medicina y de Ciencias de la Actividad Física y el Deporte, 9 (33), 51-66.

42. Olmedilla, A., Blas, A. y Laguna, M. (2010). Motivación y lesiones deportivas en jugadores de balonmano. Revista Argentina de Ciencias del Comportamiento, 2 (3), 1-7.

43. Olmedilla, A., García, C. y Martínez, F. (2006). Factores psicológicos y vulnerabilidad a las lesiones deportivas: un estudio en futbolistas. Revista de Psicología del Deporte, 15 (1), 7-19.

44. Olmedilla, A., Ortega, E., Abenza. L. y Boladeras, A. (2011). Lesiones deportivas y psicología: Una revisión (2000-2009). Cuadernos de Psicología del Deporte, 11 (1), 45-57.
45. Ortín, F.J., Montero, E., Garcés de los Fayos, J. y Olmedilla, A. (2010). Influencia de los factores psicológicos en las lesiones deportivas. Papeles del Psicólogo, 31(3), 281-288.

46. Osborn, Z., Blanton, P. y Schwebel, D. (2009). Personality and Injury Risk Among Professional Hockey Players. Journal of injury and violence research, 1 (1), 15-19.

47. OSullivan, D. M., Zuckerman, M. y Kraft, M. (1998). Personality characteristics of male and female participants in team sports. Personality and Individual Differences, 25 (1), 119- 128.

48. Pallás, J.M. y Villa, J. (2004). Métodos de investigación en clínica y epidemiología. Madrid: Elsevier.

49. Pascual, A. y Aragües, G. (1998). Lesiones deportivas y rasgos de ansiedad en los jugadores de fútbol. Medicina Clínica, 111 (2), 45-8.

50. Pérez, J. y Torrubia, R. (1986). Fiabilidad y validez de la versión española de la Escala de Búsqueda de Sensaciones (Forma V). Revista Latinoamericana de Psicología, 18 (1), 7-22.

51. Pérez, J., Ortet, G., Plá, S. y Simó, S. (1987). Escala de Búsqueda de Sensaciones para Niños y Adolescentes (EBS-J). Evaluación Psicológica, 3 (2), 283-290

52. Potgieter, J y Bisschoff, F. (1990). Sensation seeking among mediumand low-risk sports participants. Perceptual and Motor Skills, 71(3), 1203-1206.

53. Rhea, D. J. y Martin, S. (2010). Personality Trait Differences of Traditional Sport Athletes, Bullriders, and Other Alternative Sport Athletes. International Journal of Sports Science \& Coaching, 5 (1), 75-85.

54. Roberti, J. (2004). A review of behavioral and biological correlates of sensation seeking. Journal of Research in Personality, 38, 256-279.

55. Rotella, R. J. y Heyman, S. R. (1991). El estrés, las lesiones y la rehabilitación psicológica de los deportistas. En J. M. Williams (Ed.), Psicología aplicada al deporte (pp. 493-522). Madrid: Biblioteca Nueva.

56. Schroth, M. (1995). A comparison of sensation seeking among different groups of athletes and nonathletes. Personality and Individual Differences, 18 (2), 219-222.

57. Siesma, E. J., Blitvich, J. D., White, P. E. y Finch, C. F. (2011). Measuring children's self-reported sport participation, risk perception and injury history: Development and validation of a survey instrument. Journal of Science and Medicine in Sport, 14 (1), 22-26.

58. Sitkin, S. B. y Pablo, A. L. (1992). Reconceptualising the determinants of risk behavior. Academy of Management Review, 17 (1), 9-38.

59. Valliant, P. M. (1981). Personality and injury in competitive runners. Perceptual and Motor Skills, 53 (1), 251-253.

60. Van der Pligt, J. (1994). Risk appraisal and health behavior. En D. R. Rutter y L. Quine (Eds.), Social psychology and health: European perspectives. (pp. 131-151). Brookfield VT: Avebury.

61. Williams, J. M. y Andersen, M. B. (1998). Psychological Antecedents of Sport Injury: Review and Critique of the Stress and Injury Model. Journal of Applied Sport Psychology, 10 (1), 5-25.

62. Williams, J.M. (2001). Psychology of injury risk and prevention. En R.N. Singer, H.A. Hausenblas C.M. Janelle (Eds.), Handbook of sport psychology (2nd ed., pp. 766-786). New York: Wiley \& Sons.

63. Zuckerman, M. (1979). Sensation seeking: Beyond the optimal level of arousal. Hillsdale, N. J.: Lawrence Ealbaum Associates.

64. Zuckerman, M. (1983). Sensation seeking and sports. Personality and Individual Differences, 4, 285-293.

65. Zuckerman, M. (2007). Sensation seeking and risky behavior. Washington, DC: American Psychological Association.

66. Zuckerman, M., Eysenck, S.B. y Eysenck, H.J. (1978). Sensation seeking in England and America: Cross-cultural, age, and sex comparisons. Journal of Consulting and Clinical Psychology, 46 (1), 139-149. 


\section{Anexo.}

\section{Cuestionario de propensión al accidente deportivo (PAD-22)}

Edad:____ Sexo: H:__M: __ ¿Te has lesionado esta temporada? Sí__No__

\begin{tabular}{|c|c|}
\hline Lee atentamente las cuestiones y responde según la escala de valoración. & Valoración \\
\hline Valora de 1 (muy en desacuerdo) a 6 (muy de acuerdo) las siguientes cuestiones. & $1 \quad 2 \quad 3 \quad 4 \quad 5 \quad 6$ \\
\hline 1. Tengo capacidades y poderío físico para realizar cualquier actividad deportiva. & \\
\hline 2. Me gusta siempre ser el mejor y sorprender a los demás. & \\
\hline 3. Cuando pierdo me siento muy mal conmigo mismo. & \\
\hline 4. Cuando hago deporte, si no gano, no me divierto. & \\
\hline 5. Necesito superarme para ser el mejor. & \\
\hline 6. Conduzco la bicicleta sin casco. & \\
\hline 7. Hago deporte estando lesionado. & \\
\hline 8. No me importa patinar sin casco ni protecciones. & \\
\hline 9. Suelo soltarme de manos al conducir la bicicleta. & \\
\hline 10. Frecuentemente estoy mal por alguna cuestión relacionada con mi apariencia física. & \\
\hline 11. Mi cuerpo no me transmite sensaciones positivas. & \\
\hline 12. Creo que, comparado con la mayoría, mi cuerpo no parece estar en la mejor forma física. & \\
\hline 13. Me gusta tirarme desde el trampolín más alto de la piscina. & \\
\hline 14. Me gusta explorar un bosque desconocido haciendo senderismo o en bicicleta, aunque pueda perderme. & \\
\hline 15. Me gusta montarme en una montańa rusa. & \\
\hline 16. Siempre soy el primero en ofrecerme para hacer las actividades más arriesgadas. & \\
\hline 17. Algunas veces me gusta hacer cosas que impliquen pequeńos sobresaltos. & \\
\hline 18. Me gusta hacer acampada en lugares deshabitados y alejados. & \\
\hline 19. Saltar una valla no conlleva ningún peligro. & \\
\hline 20. Hacer una voltereta en el aire es poco peligroso. & \\
\hline 21. Como nado muy bien, hacer piragüismo sin chaleco-salvavidas no supone ningún riesgo para mí. & \\
\hline 22. Saltar el potro es poco peligroso. & \\
\hline
\end{tabular}

